\title{
Linking tuberous sclerosis complex, excessive mTOR signaling, and age-related neurodegeneration: a new association between TSC1 mutation and frontotemporal dementia
}

\author{
Nicholas T. Olney ${ }^{1} \cdot$ Carolina Alquezar $^{1} \cdot$ Eliana Marisa Ramos $^{2} \cdot$ Alissa L. Nana $^{1} \cdot$ Jamie C. Fong $^{1} \cdot$ \\ Anna M. Karydas ${ }^{1} \cdot$ Joanne B. Taylor $^{1} \cdot$ Melanie L. Stephens $^{1} \cdot$ Andrea R. Argouarch $^{1} \cdot$ Victoria A. Van Berlo $^{2}$. \\ Deepika R. Dokuru ${ }^{2}$ Elliott H. Sherr ${ }^{3} \cdot$ Gregory A. Jicha $^{4} \cdot$ William P. Dillon ${ }^{5} \cdot$ Rahul S. Desikan $^{5} \cdot$ Mary De May $^{1}$. \\ William W. Seeley ${ }^{1} \cdot$ Giovanni Coppola $^{2} \cdot$ Bruce L. Miller $^{1} \cdot$ Aimee W. Kao $^{1}$
}

Received: 28 June 2017 / Revised: 2 August 2017 / Accepted: 7 August 2017 / Published online: 21 August 2017

(C) The Author(s) 2017. This article is an open access publication

A 52-year-old right-handed man presented for the evaluation of progressive emotional flattening, social withdrawal, and loss of empathy. Prior to symptom onset, his wife of 21 years described him as "easy going, loving, kind, and generous." As symptoms progressed, the subject began to alienate coworkers with unwelcome and persistent practical jokes. These disinhibited behaviors were accompanied by new, compulsive Internet usage. No language, visuospatial, memory, or motor symptoms were reported.

Past medical history included chronic headaches and multiple concussions but no seizures, psychiatric disorders, or drug use. He denied cutaneous, dental, or renal abnormalities. His childhood development was

Nicholas T. Olney and Carolina Alquezar have contributed equally to this work.

Electronic supplementary material The online version of this article (doi:10.1007/s00401-017-1764-0) contains supplementary material, which is available to authorized users.

\section{Bruce L. Miller}

$\checkmark$ Aimee W. Kao

aimee.kao@ucsf.edu

1 Department of Neurology, Memory and Aging Center, University of California, Box 1207, San Francisco, CA 94158, USA

2 Department of Neurology and Department of Psychiatry, Semel Institute for Neuroscience and Human Behavior, University of California, Los Angeles, CA 90095, USA

3 Department of Neurology and Pediatrics, University of California, San Francisco, CA 94158, USA

4 Department of Neurology, Sanders-Brown Center on Aging, University of Kentucky, Lexington, KY 40536, USA

5 Department of Radiology, University of California, San Francisco, CA 94158, USA unremarkable. He completed 13 years of formal education and worked in the upper echelons of management at a professional firm.

The subject's family history (Supplementary Fig. S1a) was notable for a father with multiple concussions and late-life seizures, alcoholism, impulsiveness, and poor decision-making. An older sibling with left arm hemihypertrophy (Parkes-Weber Syndrome) was deceased after post-operative stroke. A younger sibling developed epilepsy at age 19 and underwent frontal lobe resection surgery at age 43; this individual experienced progressive difficulty with concentration and multitasking, qualifying for a diagnosis of mild cognitive impairment (MCI) in the executive domain.

On exam, the proband was 72 inches tall and weighed $220 \mathrm{lb}$ (BMI of 29.8). Skin exam revealed ungual fibromas and a shagreen patch. His neurological exam was normal except for an expansive mood, intrusive commentary, and jocularity verging on the inappropriate. Neuropsychological testing revealed impaired confrontational naming with mild deficits in semantic knowledge. Visual episodic memory, executive functioning, and information processing speed were also impaired. Other cognitive domains were within normal limits for age. Deficits localized to the right greater than left temporal and frontal lobes. Magnetic resonance imaging (MRI) revealed corresponding areas of atrophy and white matter lesions (Fig. 1a). Electroencephalogram (EEG) was negative for epileptiform activity.

The proband met criteria for probable behavioral variant frontotemporal dementia (bvFTD), a sub-type of frontotemporal lobar degeneration (FTLD), based on early behavioral disinhibition, loss of empathy, perseverative behaviors, neuropsychological profile, and MRI findings [12]. Given his family history, he was screened for known genetic causes of FTLD with no observed pathogenic variants. Therefore, the 
a
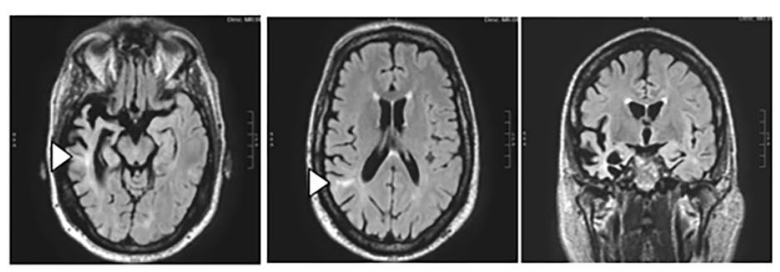

C

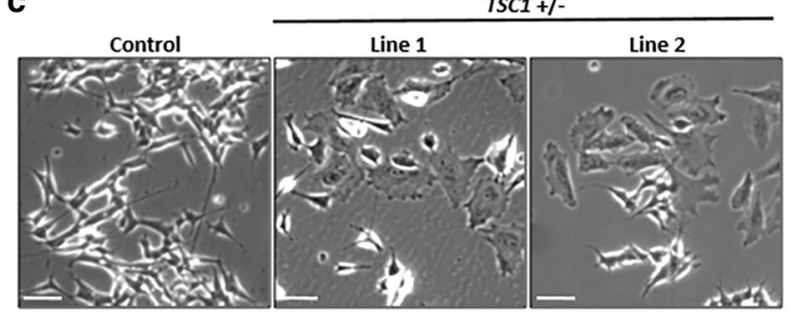

b

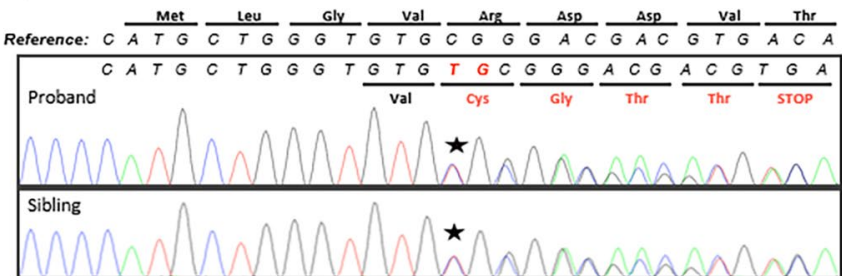

e

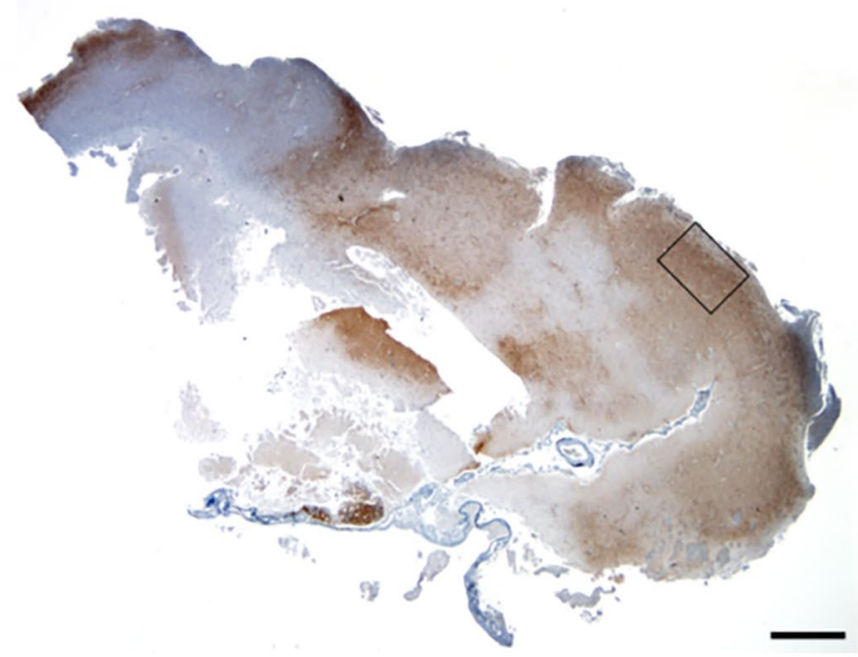

d

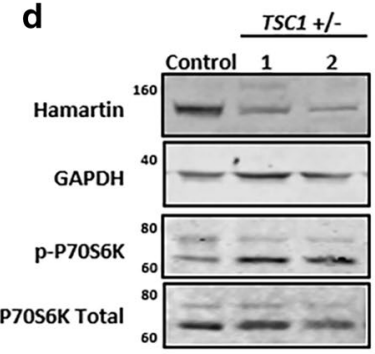

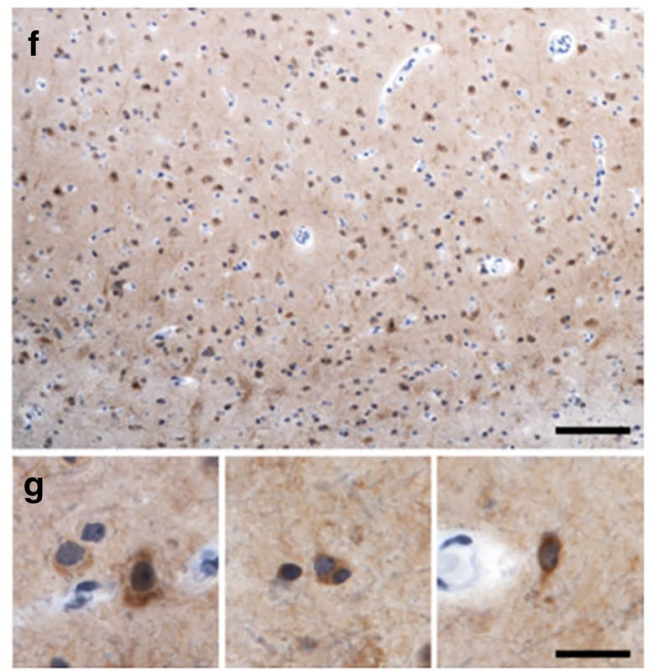

Fig. 1 Proband clinical MRI, novel TSC1 mutation, cell model of TSC1/hamartin haploinsufficiency, and neuropathology of a TSC1 mutation carrier. a Magnetic resonance imaging (MRI) FLAIR sequences revealed severe atrophy of the right anterior temporal lobe, particularly the entorhinal/perirhinal cortices and inferior temporal gyrus, as well as the hippocampus and amygdala. There was also involvement of the left anterior/mesial temporal lobe and the bilateral frontal and right parietal lobes. T2/FLAIR hyperintensities are seen within the subcortical white matter, some of which extended to the superolateral aspects of the lateral ventricles (arrowheads). These lesions can be seen in tuberous sclerosis or focal cortical dysplasias as a transmantle sign. b Sanger sequencing con- firmed a novel frameshift variant in the TSC1 gene (NM_000368.4: c.62_63insTG: p. Arg22CysfsTer5) that segregated with the proband and affected sibling. c Representative micrographs of the enlarged cell body in the undifferentiated TSC1 heterozygous mutant SHSY5Y lines. Scale bars represent 50 microns. d Representative western blots showing decreased TSC1/hamartin and increased phophoP70S6K ${ }^{\text {thr389 }}$, total tau, and phospho-tau levels in retinoic acid differentiated TSC1 +/- SH-SY5Y cells. TDP-43 levels are unchanged. eg Fragment of frontal cortex resection from the proband's sibling was received and stained for hyperphosphorylated tau, revealing patchy staining in neurons, glia, and surrounding neuropil. Scale bars represent $1000(\mathbf{e}), 250(\mathbf{f})$, and 25 (g) microns proband and his affected sibling underwent whole-exome sequencing. Among variants identified, a novel frameshift mutation in one allele of the TSC1 gene, predicted to result in early termination of the TSC1/hamartin protein, was shared by the proband and his younger sibling (Fig. 1b, Supplementary Fig. S1b, c; Table S1).

TSC1/hamartin is an upstream inhibitor of mTOR, which regulates cell growth and protein degradation [8]. 
To determine the significance of the TSCl loss of function variant, CRISPR/Cas9 genome editing [6] was used to generate a cellular model of TSC1/hamartin deficiency in SH-SY5Y cells, a human neuroblastoma cell line that can be differentiated into neuronal-like cells [7] (Supplementary Fig. S2a). We confirmed that these lines exhibited decreased TSC1/hamartin, increased cell size, and elevated P70S6K phosphorylation, consistent with mTOR over activity (Fig. 1c, Supplementary Fig. S2b, c). After retinoic acid (RA) differentiation, $T S C 1+/-$ cells maintained lower levels of TSC1/hamartin and increased mTOR activity. Interestingly, the RA-differentiated TSCl +/- lines accumulated phospho-tau and total tau. However, levels of TDP-43, another protein that aggregates in FTLD [2], were unchanged compared to control (Fig. 1d, Supplementary Fig. S2d-h).

Histological samples were obtained from the partial frontal lobe resection of the affected sibling, also a TSC1 mutation carrier. Similar to SH-SY5Y model of TSC1 heterozygosity, immunostaining of the resected tissue for phosphorylated tau revealed patchy neuropil staining as well as diffuse cytoplasmic staining of scattered neurons and glia, with variable intensity (Fig. 1e-g). No neurofibrillary tangles, Pick bodies, glial inclusions, or other features of an inherited or sporadic tauopathy were observed. Sections were also stained for TDP-43, phospho-TDP-43, amyloid beta $(A \beta), \alpha$-synuclein, and ubiquitin. Surprisingly, they did not demonstrate elevated immunoreactivity for any other neurodegenerative disease proteins (data not shown).

In this case report, we describe the first case of bvFTD in an adult with sub-clinical tuberous sclerosis complex (TSC) due to a novel TSCl frameshift variant. Although TSC has been called an "infantile tauopathy" [13], TSC1 mutations have not previously been implicated in age-associated neurodegenerative disease. This report, therefore, potentially adds $T S C 1$ to the list of genes that are implicated in both a juvenile-onset lysosomal storage disease and adult-onset neurodegeneration. Others include $G B A$ and $A T P 13 A 2$ in Parkinson's disease, CTSD in Alzheimer's disease, and PGRN in FTLD [10, 15]. The TSC1/ TSC2 complex normally inhibits mTOR activity, with loss of function mutations resulting in overactive mTOR and consequential increased protein synthesis and decreased protein degradation $[3,8,9]$. Strikingly, the decreased protein degradation seems selective for tau, as evidenced from both our cell-based and neuropathological data. This case now suggests that with age, accumulated tau can precipitate neuron loss and neurodegeneration. It also raises the intriguing possibility that tau metabolism is selectively regulated by the mTOR pathway. Our data suggest that TSCI loss of heterozygosity, which is necessary for TSC-related tumors [11], is not required for TSC1-related tau accumulation and neurodegeneration.
Our findings could be highly significant to the care of TSC patients. Many features of tuberous sclerosis associated neuropsychiatric disorders (TAND) [4, 5], including obsessive behavior, attention deficits, altered eating, impulsivity, memory loss, and language dysfunction, overlap with bvFTD criteria. Thus, these two clinical entities may represent overlapping disorders precipitated by progressive tau pathology. Additional sequencing of the TSC1/2 genes in FTLD cohorts, use of new tau-based PET imaging, and review of neuropathological specimens from older TSC patients may further support the association between TSC, TAND, and FTLD.

This study extends the relationship between mTOR and tau metabolism $[1,16]$ to include a potential age-related component. How does aging alter the TSC $1 / \mathrm{mTOR}$ axis to potentially contribute to the development of neurodegeneration? Why do tau and phospho-tau accumulate, while related proteins that undergo chaperone mediated autophagy, such as TDP-43, do not? Do TSC2 mutations also link to age-related tauopathies? These questions and others are stimulated by our report. In the meantime, this study supports trials of rapamycin analogs which are already underway for neurodegeneration [14], and moves us towards a personalized medicine future in which subpopulations of tauopathy patients may be selectively responsive to this therapeutic approach.

Acknowledgements This work is supported by the Rainwater Tau Consortium and NIH/NIA (P50AG023501, P01AG019724, T32AG023481-11S1, and P50 AG1657303). Additional support was provided by the Ramón Areces Foundation (C.A.), the Paul G. Allen Family Foundation (A.W.K.), NIH/NIA RC1 AG035610 (G.C.), John Douglas French Alzheimer's Foundation (G.C.), National Alzheimer's Coordinating Center Junior Investigator Award (R.S.D.), ASNR Alzheimer's Imaging Research Grant Program (R.S.D.), RSNA Resident Fellow grant (R.S.D.), and NIH/NIDA grant U24DA041123 (R.S.D.). We thank Thomas Pittman and William Markesbury from the University of Kentucky for sharing the pathological specimens. We thank the Tau Consortium Stem Cell group for technical advice and support.

Author contributions A.W.K., B.L.M, G.C., and W.W.S designed the study. N.T.O., C.A., E.M.R, A.L.N., J.C.F., A.M.K., J.B.T., M.L.S., M.D.D., A.R.A., V.A.v.B., D.R.D., E.H.S, G.A.J., W.P.D., R.S.D., B.L.M., A.W.K., G.C., and W.W.S. were responsible for data acquisition and analysis. N.T.O., C.A., E.M.R., J.C.F., J.B.T., R.S.D., and A.N.L. created the figures. N.T.O., C.A., W.W.S, G.C., B.L.M., and A.W.K. wrote the manuscript.

\section{Compliance with ethical standards}

Conflict of interest Dr. Seeley reports personal fees from Merck, Inc., personal fees from Bristol Myers-Squibb, outside the submitted work. Dr. Miller has served as an Advisor/Director to The Tau Consortium, The John Douglas French Foundation, The Larry L. Hillblom Foundation, Medical Advisory Board, National Institute for Health Research, Cambridge Biomedical Research Centre and its subunit, the Biomedical Research Unit in Dementia (UK); he has served as an External Advisor to University of Washington ADRC, Stanford University 
ADRC, and University of Pittsburgh ADRC; he receives royalties from Cambridge University Press, Guilford Publications, Inc., and Neurocase. All other authors have no conflicts of interest to report.

Open Access This article is distributed under the terms of the Creative Commons Attribution 4.0 International License (http://creativecommons.org/licenses/by/4.0/), which permits unrestricted use, distribution, and reproduction in any medium, provided you give appropriate credit to the original author(s) and the source, provide a link to the Creative Commons license, and indicate if changes were made.

\section{References}

1. Caccamo A, Magri A, Medina DX, Wisely EV, Lopez-Aranda MF, Silva AJ, Oddo S (2013) mTOR regulates tau phosphorylation and degradation: implications for Alzheimer's disease and other tauopathies. Aging Cell 12:370-380. doi:10.1111/ acel.12057

2. Cairns NJ, Bigio EH, Mackenzie IR, Neumann M, Lee VM, Hatanpaa KJ, White CL 3rd, Schneider JA, Grinberg LT, Halliday $G$ et al (2007) Neuropathologic diagnostic and nosologic criteria for frontotemporal lobar degeneration: consensus of the Consortium for Frontotemporal Lobar Degeneration. Acta Neuropathol (Berl) 114:5-22

3. Crino PB (2016) The mTOR signalling cascade: paving new roads to cure neurological disease. Nat Rev Neurol 12:379-392. doi:10.1038/nrneurol.2016.81

4. Curatolo P, Moavero R, de Vries PJ (2015) Neurological and neuropsychiatric aspects of tuberous sclerosis complex. Lancet Neurol 14:733-745. doi:10.1016/S1474-4422(15)00069-1

5. de Vries PJ, Whittemore VH, Leclezio L, Byars AW, Dunn D, Ess KC, Hook D, King BH, Sahin M, Jansen A (2015) Tuberous sclerosis associated neuropsychiatric disorders (TAND) and the TAND Checklist. Pediatr Neurol 52:25-35. doi:10.1016/j. pediatrneurol.2014.10.004

6. Doudna JA, Charpentier E (2014) Genome editing. The new frontier of genome engineering with CRISPR-Cas9. Science 346:1258096. doi:10.1126/science. 1258096
7. Encinas M, Iglesias M, Liu Y, Wang H, Muhaisen A, Cena V, Gallego C, Comella JX (2000) Sequential treatment of SHSY5Y cells with retinoic acid and brain-derived neurotrophic factor gives rise to fully differentiated, neurotrophic factordependent, human neuron-like cells. J Neurochem 75:991-1003

8. Laplante M, Sabatini DM (2012) mTOR signaling in growth control and disease. Cell 149:274-293. doi:10.1016/j. cell.2012.03.017

9. Lipton JO, Sahin M (2014) The neurology of mTOR. Neuron 84:275-291. doi:10.1016/j.neuron.2014.09.034

10. Nixon RA (2013) The role of autophagy in neurodegenerative disease. Nat Med 19:983-997. doi:10.1038/nm.3232

11. Qin W, Chan JA, Vinters HV, Mathern GW, Franz DN, Taillon BE, Bouffard P, Kwiatkowski DJ (2010) Analysis of TSC cortical tubers by deep sequencing of TSC1, TSC2 and KRAS demonstrates that small second-hit mutations in these genes are rare events. Brain Pathol 20:1096-1105. doi:10.1111/j.1750-3639.2010.00416.x

12. Rascovsky K, Hodges JR, Knopman D, Mendez MF, Kramer JH, Neuhaus J, van Swieten JC, Seelaar H, Dopper EG, Onyike $\mathrm{CU}$ et al (2011) Sensitivity of revised diagnostic criteria for the behavioural variant of frontotemporal dementia. Brain 134:2456-2477. doi:10.1093/brain/awr179

13. Sarnat HB, Flores-Sarnat L (2015) Infantile tauopathies: hemimegalencephaly; tuberous sclerosis complex; focal cortical dysplasia 2; ganglioglioma. Brain Dev 37:553-562. doi:10.1016/j. braindev.2014.08.010

14. Siman R, Cocca R, Dong Y (2015) The mTOR inhibitor rapamycin mitigates perforant pathway neurodegeneration and synapse loss in a mouse model of early-stage alzheimer-type tauopathy. PLoS ONE 10:e0142340. doi:10.1371/journal.pone.0142340

15. Smith KR, Damiano J, Franceschetti S, Carpenter S, Canafoglia L, Morbin M, Rossi G, Pareyson D, Mole SE, Staropoli JF et al (2012) Strikingly different clinicopathological phenotypes determined by progranulin-mutation dosage. Am J Hum Genet 90:1102-1107. doi:10.1016/j.ajhg.2012.04.021

16. Tang Z, Ioja E, Bereczki E, Hultenby K, Li C, Guan Z, Winblad B, Pei JJ (2015) mTor mediates tau localization and secretion: implication for Alzheimer's disease. Biochim Biophys Acta 1853:1646-1657. doi:10.1016/j.bbamcr.2015.03.003 